\title{
Collapse of a hockey player: ruptured aneurysm of the renal artery
}

\section{Gavin W. Smart MD, John S. Crowley MB, Denis J. Lavoie MD}

Previously published at www.cmaj.ca

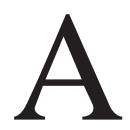

10-year-old male novice hockey player was skating backward during a routine practice when he fell across a teammate's hockey stick, which hit his left flank. The player collapsed immediately, vomited and complained of severe left back pain. He was transferred by ambulance to hospital, where he arrived 30 minutes postinjury.

On initial assessment, his blood pressure was $78 / 50 \mathrm{~mm} \mathrm{Hg}$, heart rate was 120 beats/min, Glasgow Coma Scale score was 13 , and he had tenderness in the left upper quadrant and flank. Despite aggressive fluid resuscitation, the patient became unresponsive and suffered respiratory arrest ten minutes after arrival, necessitating intubation. Initial hemoglobin was 95 (normal 140-175) g/L. The differential diagnosis was splenic rupture with intraperitoneal hemorrhage or renal laceration with retroperitoneal hemorrhage.

At emergency laparotomy about 90 minutes after the injury, the surgeons noted that the liver and spleen were normal, but there was a large expanding left retroperitoneal hematoma with only $3 \mathrm{~mL}$ of intraperitoneal blood. The patient's hemodynamic status remained precarious, despite vigorous red blood cell and crystalloid infusion with vasopressor support.

Limited intravenous pyelography, performed on the operating table, showed no function within the left kidney with loss of normal perinephric landmarks, suggesting catastrophic injury. After cutting off the blood supply to the left kidney, blood pressure stabilized promptly. During left nephrectomy, a large volume of free blood and clot was found in the retroperitoneal space. On preliminary inspection, the resected kidney appeared normal. The patient was transferred to the recovery room in stable condition. He was discharged five days after surgery and he recovered uneventfully.

Sectioning of the left kidney revealed a $2.5-\mathrm{cm}$ aneurysm, probably of congenital origin, (Figure 1) with a 1-cm full thickness laceration within the distal renal artery. There was evidence of previous rupture and formation of a pseudoaneurysm. Subsequent magnetic resonance angiography showed no other vascular anomaly.

From the Departments of Family Practice and Surgery, Vernon Jubilee Hospital, Vernon, BC

CMAJ 2010. DOI:10.1503/cmaj.090766

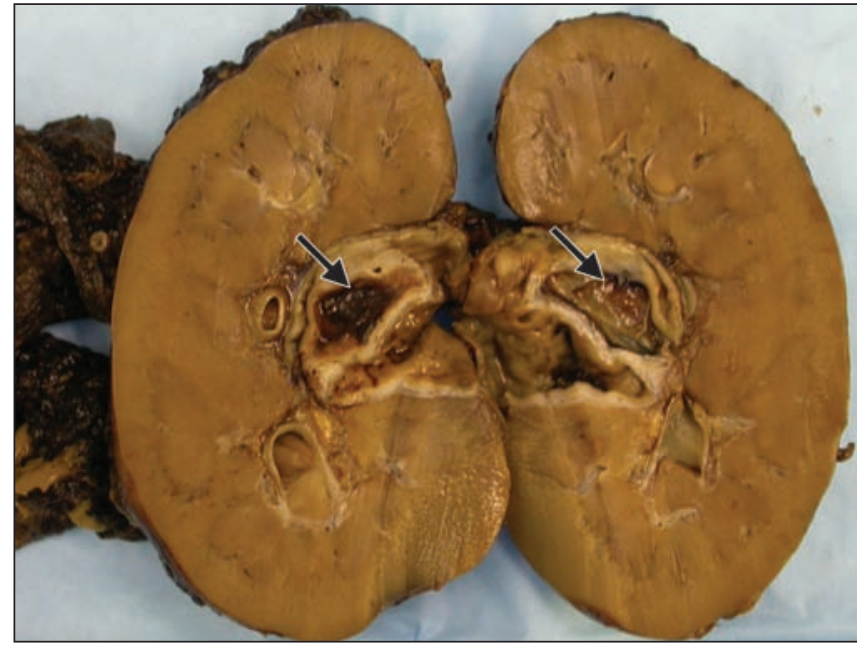

Figure 1: Cross-section of the resected kidney of a 10-year-old boy, showing aneurysmal dilation within the distal renal artery (arrows).

Aneurysms of the visceral arteries are rare but potentially life-threatening anomalies. ${ }^{1}$ Aneurysms of the splenic and renal arteries account for most cases in published series. They are usually asymptomatic, but rupture can result in death. ${ }^{2}$ Aneurysms of the renal artery occur with an estimated prevalence of $0.6 \%$ to $1.0 \%$ angiographically and are bilateral in about $10 \%$ of cases. ${ }^{3}$ With the widespread use of abdominal imaging modalities, detection of asymptomatic aneurysms of the renal artery is more common. No consensus exists regarding the optimal management of this finding. The two most common elective interventions are resection of the aneurysm with reconstruction of the renal artery, and endovascular embolization.

This article has been peer reviewed.

Competing interests: None declared.

\section{REFERENCES}

1. Kalko Y, Ugurlucan M, Basaran M, et al. Visceral artery aneurysms. Heart Surg Forum 2007; 10:E24-9.

2. Zelenock GB, Stanley JC. Spanchnic artery aneurysms. In: Rutherford RB, editor. Vascular surgery. 5th ed., Philadelphia (PA): WB Saunders; 2000: 1369-82.

3. Pfeiffer T, Reiher L, Grabitz K, et al. Reconstruction for renal artery aneurysm: operative techniques and long-term results. J Vasc Surg 2003;37:293-300. 